\title{
Hyperspectral Imaging of Photonic Cellulose Nanocrystal Films: Structure of Local Defects and Implications for Self-Assembly Pathways
}

\author{
Bonan Zhu, Villads E. Johansen, Gen Kamita, Giulia Guidetti, Mélanie M. Bay, Thomas G. Parton, \\ Bruno Frka-Petesic,* and Silvia Vignolini*
}

Cite This: ACS Nano 2020, 14, 15361-15373

Read Online

\section{ACCESS \\ 山lll Metrics \& More \\ Article Recommendations \\ Supporting Information}

ABSTRACT: Cellulose nanocrystals (CNCs) can spontaneously assemble into chiral nematic films capable of reflecting circularly polarized light in the visible range. As many other photonic materials obtained by bottom-up approaches, CNC films often display defects that greatly impact their visual appearance. Here, we study the optical response of defects in photonic CNC films, coupling optical microscopy with hyperspectral imaging, and we compare it to optical simulations of discontinuous cholesteric structures of increasing complexity. Cross-sectional SEM observations of the film structure guided

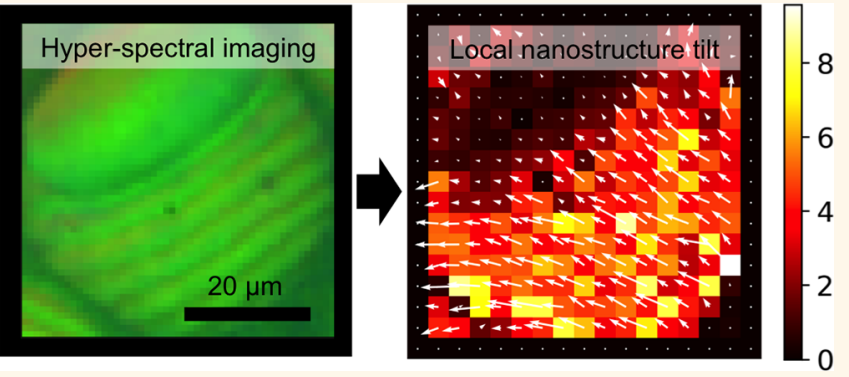
the choice of simulation parameters and showed excellent agreement with experimental optical patterns. More importantly, it strongly suggests that the last fraction of CNCs to selfassemble, upon solvent evaporation, does not undergo the typical nucleation and growth pathway, but a spinodal decomposition, an alternative self-assembly pathway so far overlooked in cast films and that can have far-reaching consequences on choices of CNC sources and assembly conditions.

KEYWORDS: cellulose nanocrystals, chiral nematic defects, hyperspectral imaging, security printing, nucleation and growth, spinodal decomposition

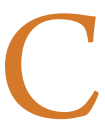
ellulose nanocrystals (CNCs) are splinter-like chiral nanorods able to organize into cholesteric suspensions. After solvent evaporation, they can produce photonic films with a broad range of optical properties, ${ }^{1,2}$ with applications demonstrated over the last two decades. ${ }^{3,4}$ The effective optical response of these films and their resulting visual appearance are strongly influenced by the degree of alignment and order (or disorder) from the microscale to the macroscopic scale. ${ }^{5-9}$ In practice, various defects are commonly found in CNC films and certainly play an essential role in generating the variety of different macroscopic visual aspects they present. ${ }^{6,10,11}$ However, the lack of optical analysis of defects or simulation of the optical response of defect structures in CNC films has limited our understanding of how the presence of these defects determines the final optical response. $^{12}$

Recent investigations of photonic films made from CNCs have emphasized the importance of the self-assembly pathway leading to the final structure and its resulting visual appearance. $^{2,5,8}$ When a CNC suspension is cast on a flat substrate or in a dish and allowed to dry, the volume fraction of $\mathrm{CNC}$ gradually increases, favoring the phase transition from an isotropic to a cholesteric liquid crystalline phase. This order is locally characterized by its pitch, $p$, typically in the micron range, and its helical axis, $\mathbf{m}$. The cholesteric phase typically forms by a nucleation and growth (NG) mechanism, whereby cholesteric droplets, or "tactoids", ${ }^{13}$ form in coexistence with the isotropic phase. The drying suspension approaches thermodynamic equilibrium through tactoid growth, coalescence, and sedimentation of the denser cholesteric phase. ${ }^{14}$ Although planar anchoring of the cholesteric phase is favored at both liquid-substrate and liquid-air interfaces, ${ }^{15,16}$ further solvent evaporation induces kinetic arrest of the suspension into a soft solid, ${ }^{17-19}$ preventing further equilibration. The arrested photonic structure then undergoes anisotropic shrinkage as the solvent completely evaporates, compressing

Received: July 11, 2020

Accepted: October 13, 2020

Published: October 22, 2020

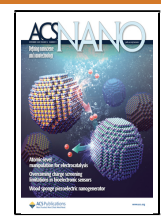



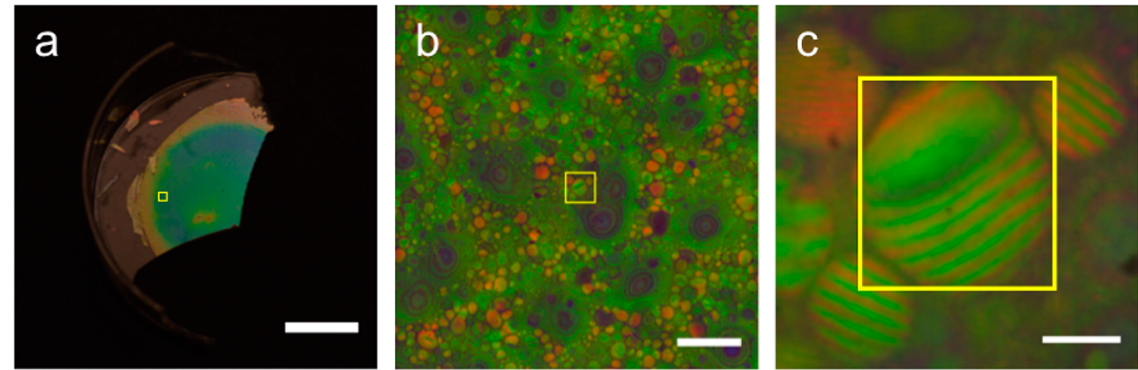

Figure 1. Observation of a CNC photonic film at different length scales. (a) Macroscopic photograph of the film attached to the Petri dish substrate (scale bar $1 \mathrm{~cm}$ ). Note that the film was fractured for cross-sectional SEM imaging. (b) Optical microscopy observed in reflection through a LCP filter using the same high-magnification objective $(50 \times, \mathrm{NA}=0.4)$ and obtained by stitching 121 photos to image a large area (scale bar $200 \mu \mathrm{m}$, higher resolution available in Figure S1). (c) Detail of (b) showing individual domains with fringes (scale bar $20 \mu \mathrm{m}$ ). The region of interest in $(b)$ and $(c)$ is indicated by yellow squares.
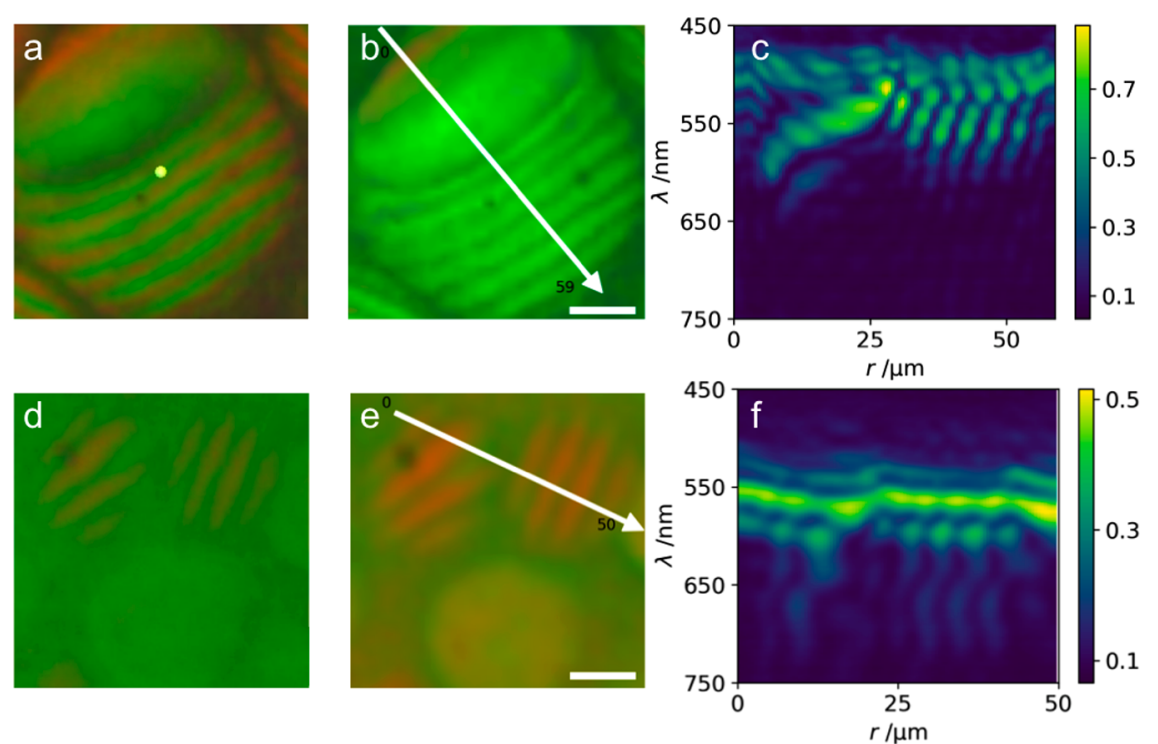

Figure 2. Hyperspectral imaging of a typical defect. Optical microscopy images obtained in reflection through an LCP filter of the same domain as in Figure 1c: (a) with the microscope camera and (b) from the HSI scan after integration into its RGB components. The light dot in the center of (a) indicates the spot size of the scanning fiber, resulting in a resolution about $2 \mu \mathrm{m}$ (the scanning step is $1 \mu \mathrm{m}$ ). (c) Spectra measured along the arrow in (b), revealing asymmetric cyclic shifts of reflection peaks in the stripy pattern region. (d-f) Another example of defect analysis similar to $(a-c)$.

the cholesteric pitch into the submicron range and allowing for strong, left-circular polarized optical reflections of specific visible wavelengths. ${ }^{5,7,8,20}$ While this general description can account for the overall optical response of films on the macroscopic level, structural analysis of local defects can reveal more subtle phenomena occurring in the self-assembly process.

Hyperspectral imaging (HSI) is an optical sample characterization technique in which the optical response is detected over many spectral bands for each pixel within the image, as opposed to the more common trichromatic (RGB) imaging performed by a typical color camera. While originally developed for astronomy, this technique was gradually developed in other sciences, fundamental and applied, ranging from food inspection, forensic document examination, medical surgery and diagnostics, defense and homeland security applications, precision agriculture and water management, material identification, and mapping of artwork. ${ }^{21}$ In colloidal, material, and life sciences, UV-vis-NIR HSI has emerged as an excellent tool to discriminate multiple chemical species, particularly when their emission or reflectance spectra are partially overlapping (fluorescent proteins, quantum dots, organic fluorophores, etc.). ${ }^{22}$ Likewise, this tool is also well adapted to easily characterize the spectral signature of photonic nanostructures with local structural variations or defects. ${ }^{23,24}$

In this work, we study the optical response of defects in photonic CNC films, coupling optical microscopy with hyperspectral imaging, and we compare it to optical simulations of discontinuous cholesteric structures of increasing complexity. Cross-sectional SEM observations of the film structure guided the choice of simulation parameters and showed excellent agreement with experimental optical patterns. Since the formation of CNC films involves a succession of complex self-assembly steps that all participate in shaping the observed defects, the optical signature of these defects can be decoded to retrieve information about the microstructure and better understand the whole self-assembly process. ${ }^{2,25} \mathrm{We}$ conclude the structural analysis of the defect with a revisited understanding of the CNC self-assembly pathways, suggesting the existence of two different phase transition processes, namely an early NG phase followed by a later spinodal decomposition (SD) of the remaining CNCs. 


\section{RESULTS AND DISCUSSION}

Optical Microscopy and Hyperspectral Imaging. Figure 1 shows a macroscopic photograph as well as optical microscopy images of a film produced by self-assembly, whereby an aqueous suspension of CNC was allowed to dry in a Petri dish (details in Experimental Methods). In this example, the film color is inhomogeneous, with clear redshift near the edge as observed in many other examples in the literature. ${ }^{6,26,27}$ In the center of the sample, optical microscopy images were captured in reflection through a left-circular polarization (LCP) filter using high magnification (50X objective) and a color CCD camera (Figure $1 \mathrm{~b}$ ). A scanning procedure followed by the stitching of 121 images with ImageJ allowed for a large-scale overview of about $1 \mathrm{~mm}^{2}$ with high resolution. The acquisition of high-resolution images on a large area (all images are taken with same magnification, numerical aperture, and illumination conditions) guaranteed a direct comparison of the macroscopic and microscopic images without artifacts leading to color change. The large-scale imaging revealed many defects, associated with blue, green, and red regions. A closer look at one particular defect is illustrated in Figure 1c and presents a clear stripy pattern, reminiscent of a tilted tactoid trapped within the film. ${ }^{14}$ This periodicity is however in the micron range, which is much bigger than the pitch required to reflect the light in the visible domain, and therefore it cannot be simply interpreted as the pitch. Alternatively, another type of structure encountered in the film is a set of concentric blue and green rings, which can be originated from tactoids initially oriented rather vertically $(\mathbf{m} / / \mathbf{z}$, see white square in Figure $S 1)$.

While regular optical microscopy images captured with a color camera provide sharp spatial resolution at the micron scale, they also provide limited spectral resolution because the signal is integrated only into three wavelength channels (RGB). To improve the spectral resolution, we performed a HSI analysis at the microscopic scale using hyperspectral scanning microscopy implemented via point-scanning spectrometry, as illustrated in Figure $2 .^{22}$ In practice, an optical fiber coupled to a spectrometer was conjugated with the focal plane of the objective (see schematic of the experimental setup in Figure S2) so as to collect light only from a small area of the field of view of the objective (the collection spot has diameter of $\varnothing \sim 2 \mu \mathrm{m}$, which defines the spatial resolution of the image, and is indicated with a light spot in Figure 2a). HSI datacubes of coordinates $(x, y, \lambda)$ were obtained by scanning the sample in the $(\mathbf{x}, \mathbf{y})$ plane with steps of $1 \mu \mathrm{m}$ in each direction and for each spatial position collecting a spectra resolved in wavelength $(\lambda)$. By integrating the spectral information, it is therefore possible to reconstruct RGB images and verify them against the initial RGB images captured by the camera. To do so, the hyperspectral images were obtained simply by integrating the spectral components pixel by pixel with the CIE standard observers (details in Supporting Information). ${ }^{28}$ The reconstructed hyperspectral image captures the salient features of the regions of interest (Figure $2 \mathrm{~b}, 51 \times 51$ pixels of $1 \times 1 \mu \mathrm{m}^{2}$ each) despite having lower spatial resolution than conventional digital photography with the color camera, validating our experimental setup. More importantly, the HSI data enable plotting of spectral information along a specific direction, as illustrated in Figure $2 \mathrm{a}-\mathrm{c}$. LCP reflectance spectra collected along the stripy pattern of alternating bands in Figure $2 \mathrm{a}$ (indicated by a white arrow in Figure $2 b$ ) are shown as an intensity heatmap in Figure 2c. Interestingly, the heatmap exhibits specific asymmetric oscillations in the positions of spectral peaks along the scanning direction. This optical response was also observed elsewhere in the film. Figure $2 \mathrm{~d}-\mathrm{f}$ shows the same analysis applied to another region of interest as a further example. Complementary microscope images and HSI scans using RCP light are reported in Figures S3 and S4. There, the signal in RCP is almost negligible compared to LCP, but a similar stripy pattern is recognizable (with higher RCP values often corresponding to lower LCP ones, and vice versa).

Optical Response Simulation of Simple Defects. To investigate the effect of defects in the helicoidal CNC arrangement, we analyzed simulated structures at several levels of complexity. We selected structures based on their ability to provide clear demonstrations of the roles of different defect features, rather than their likelihood of emergence from CNC self-assembly.

Optical analysis of simulated defects was implemented using a transfer matrix method based on existing formalisms. ${ }^{29}$ In the transfer matrix method, the helicoidal CNC arrangement is treated as a stack of thin uniaxial anisotropic crystals slabs whose optical axes follow the nematic directors and rotate in plane. Refractive indices within the CNC film were taken from the literature with extraordinary and ordinary rays as $n_{\mathrm{e}}=1.586$ and $n_{\mathrm{o}}=1.524$, respectively, with average refractive index $n=$ $\left(n_{\mathrm{e}}+n_{\mathrm{o}}\right) / 2$. $^{6}$ The top and bottom half spaces were set to air $\left(n_{\text {air }}=1\right)$ and polystyrene $\left(n_{\mathrm{ps}}=1.55\right.$, birefringence neglected $)$ respectively.

Domain Discontinuity. In the simplest case, a onedimensional discontinuity in the helical stack is introduced between two left-handed cholesteric domains of identical pitch $p_{1}$ and same vertical orientation $(\mathbf{m} / / \mathbf{z})$ stacked above one another. In this case, the defect consists in an abrupt rotation of an angle $\varphi$ of the director field $\mathbf{n}$ at the interface between the top and bottom regions (Figure $3 \mathrm{~b}$ ). We defined the (xyz) framework as right-handed, with the $\mathbf{z}$ axis pointing downward and the incident light propagating toward positive $z$ values. Under such definition, the angle $\varphi$ was thus defined positive in the counterclockwise direction when viewed from below and
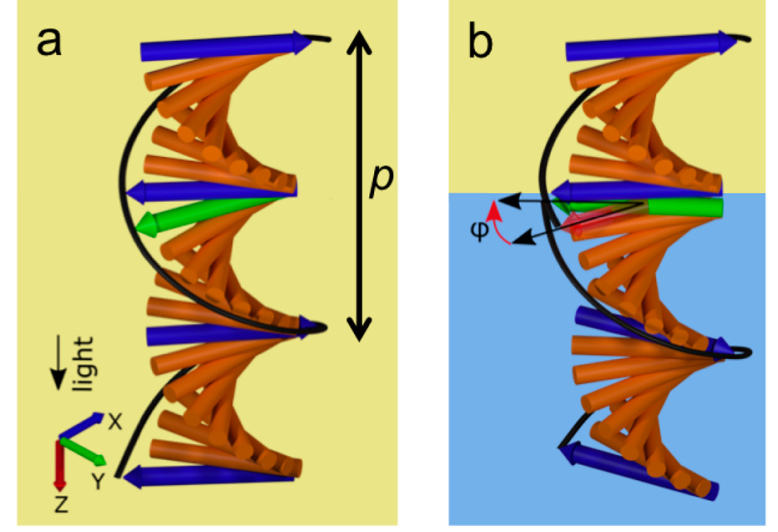

Figure 3. (a) Schematic of a defect-free helicoidal structure. Each cylinder represents the orientation of the nanocrystals in the infinitesimal layer. The nematic director is traced by the black curve and the apparent half-pitch $p / 2$ is indicated by the separation between the blue rods. (b) A defective structure characterized by a relative azimuthal rotation $\varphi$ of the bottom region with respect to the top region. 

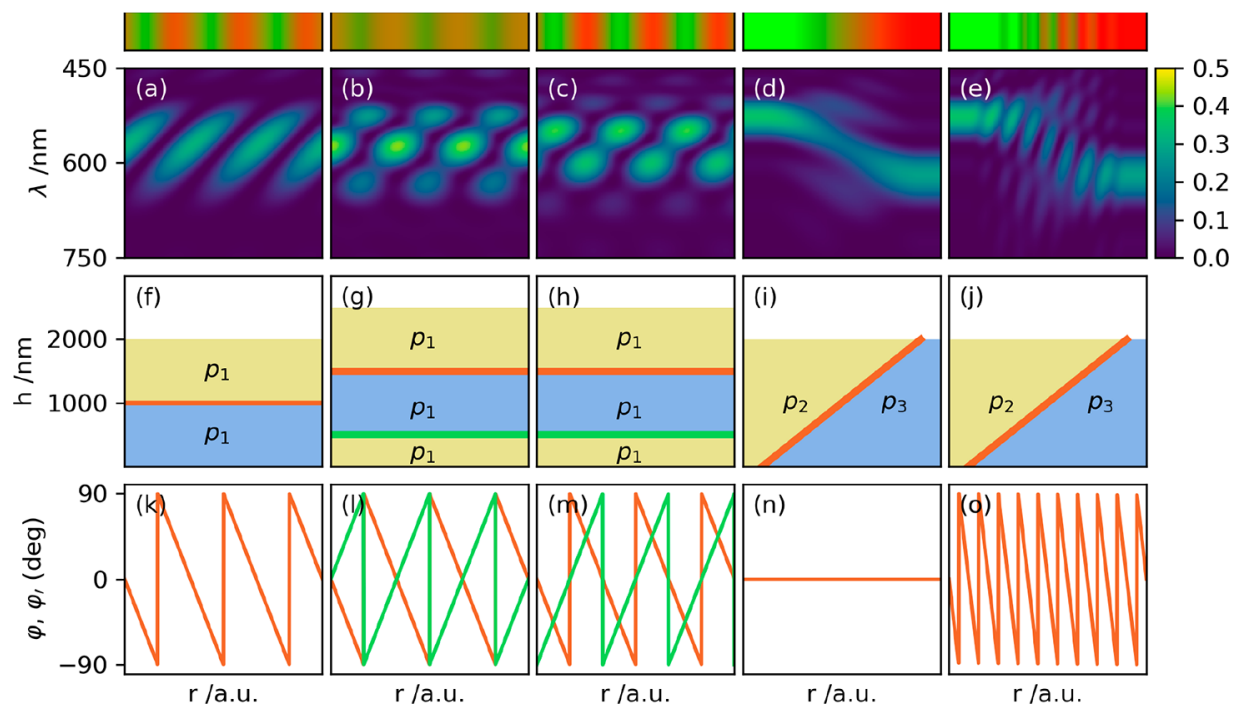

Figure 4. Simulation of reflection spectra arising from defects at boundaries between cholesteric domains (the headers represent the resulting color patterns). ( $(a-e)$ Intensity of simulated spectra reported in function of wavelength $(\lambda)$ and position $(r)$. ( $f-j)$ Corresponding defect structure, using $p_{1}=370 \mathrm{~nm}, p_{2}=340 \mathrm{~nm}$, and $p_{3}=400 \mathrm{~nm}$ and angular mismatches $\varphi$ (at orange interface) and $\varphi^{\prime}$ (at green interface). ( $\mathrm{k}-\mathrm{o}$ ) Angular mismatches $\varphi$ (orange) and $\varphi^{\prime}$ (green) against position $r$, reported here in arbitrary units. Corresponding colors are shown on top of the spectra $(\mathrm{a}-\mathrm{e})$. Averaged spectra across the whole defect area are available in Figure S5.

clockwise in top view. At the junction, the helix defect locally increases the winding of the helical structure for $0^{\circ}<\varphi<90^{\circ}$ and decreases the winding for $-90^{\circ}<\varphi<0^{\circ}$, while for $\varphi=0^{\circ}$ modulo $180^{\circ}$, the defect vanishes and the two regions behave as a single homogeneous domain. In Figure 4, column 1 (a, f, and $\mathrm{k}$ ), the reflection spectra of the LCP component are reported as a function of an arbitrary distance $r$ over which the angle $\varphi$ cyclically varies at the junction of two cholesteric domains of constant thickness $h=1000 \mathrm{~nm}$ and identical pitches $p_{1}=370 \mathrm{~nm}$. The resulting simulated spectra reproduce the asymmetric oscillations with $180^{\circ}$ periodicity seen in the experimental spectra. Importantly, the simulated spectra show a redshift as $\varphi$ is progressively increased, which is consistent with a local unwinding of the helicoidal stack at the junction between the two stacks. The spectra are also consistent with a two-color stripe periodicity corresponding to $\Delta$, the half-period of the modulation of the angle $\varphi$ along the distance $r$ (see Figures 2 and 5). It could be informative to relate this characteristic optical signature to the existence of a cyclic modulation of the Berry phase between the reflected light beams by the two cholesteric domains. ${ }^{30}$ An averaged spectrum obtained by integration over the defect region (Figure S5a) shows that the width of the averaged spectral peak is about twice as large as the peak width for the corresponding monodomain, even though the two domains have the same pitch. The high sensitivity of the optical response to defects decreases however with the domain thickness, as illustrated in Figure S6 when using $h=20 \mu \mathrm{m}$ instead of $h=1 \mu \mathrm{m}$. The broadening of the spectrum compared to the idealized structure is still present after averaging over the periodic defect, but its color pattern fades drastically, especially as the defect is localized further away from the top air-film interface.

The one-dimensional two-domain defect model captures some essential features of experimentally observed defects but does not fully explain their complex optical response. We therefore added a further layer of complexity to our model by introducing an additional third cholesteric domain with the
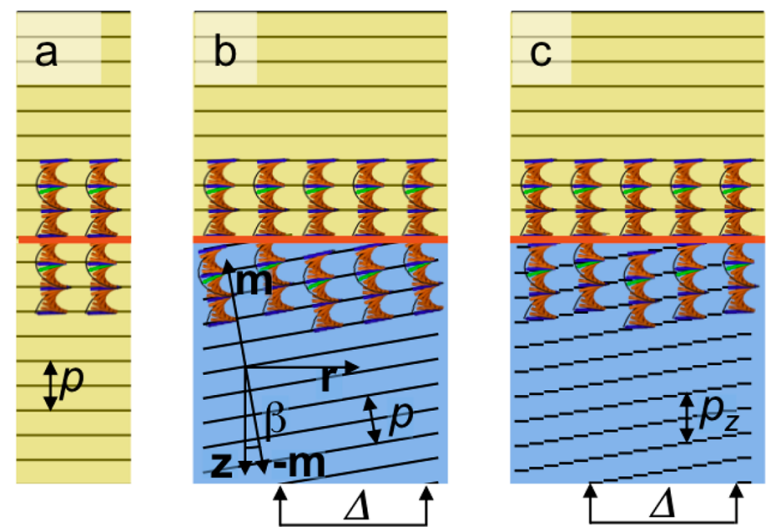

Figure 5. Schematic representation of the cross-section of a stripy defect caused by a tilt of the bottom domain. (a) In absence of tilt (i.e., helix axis $\mathrm{m}$ being vertical), the structure corresponds to one single domain. (b) The tilt of the bottom domain by a small angle $\beta$ induces a periodic modulation of the phase mismatch $\varphi(r)=\pi r /$ $\Delta$, whereby the lateral periodicity is $\Delta=p /(2 \sin \beta)=p_{z} /(2 \tan \beta)$. (c) To simulate the optical response, this structure can be approximated for small enough tilts $\beta$ to two vertically aligned domains, where the bottom pitch is $p_{z}=p / \cos \beta \approx p$ and is locally rotated about the axis by the corresponding phase $\varphi(r)$.

same pitch $p_{1}$ and a thickness of $500 \mathrm{~nm}$. In Figure 4, column 2 (b, g, and 1$)$, the helical stack of the domain 3 was set to be in phase with the domain 1 . As a result, the angle $\varphi$ at the junction between domains 1 and 2 led to an exactly opposite mismatch phase $\varphi^{\prime}=-\varphi$ between the domains 2 and 3 . In this case, the addition of an extra domain led to additional oscillations, similar to those reported experimentally in Figure 2c. These additional oscillations can be interpreted as superimposed oscillations of opposite directions, in agreement with increasing values of $\varphi$ (orange lines) but decreasing values of $\varphi^{\prime}$ (green lines) as $r$ increases. The averaged spectra obtained by integration over the defect (Figure S5b) reveal a primary peak at the same wavelength as a monodomain structure, with two secondary peaks at higher and lower 
wavelengths with higher reflectance than the secondary peaks expected for a defect-free cholesteric structure.

Interestingly, when the helical stack in the cholesteric domain 3 was shifted by a rotation of $+90^{\circ}$ (modulo $180^{\circ}$ ) with respect to the domain 1 (i.e., $\varphi^{\prime}=90^{\circ}-\varphi$ ), two main peaks of comparable intensity were found, centered at higher and lower wavelengths than the one expected in a monodomain and alternating periodically. These two peaks are also clearly seen in the averaged spectra (Figure S5b). The spatial position where the peaks are found is shifted laterally on the $r$ axis by $-\Delta / 4$ for the red-shifted peak and $+\Delta / 4$ for the blue-shifted one (Figure 4, column $3 \mathrm{c}, \mathrm{h}$, and j), thus maintaining a distance of $\Delta / 2$ between them. These two opposite color shifts are consistent with a matching of periodic unwinding $\left(\varphi=\varphi^{\prime}=45^{\circ}\right)$ or overwinding $\left(\varphi=\varphi^{\prime}=-45^{\circ}\right)$ of the two defect regions over a period $\Delta$ (i.e., for $\varphi=$ $\left.\left[-90^{\circ}, 90^{\circ}\right]\right)$. In this two-peak defect configuration, the resulting RGB image produced stripes of much sharper green and red hues than in the previous, less color-saturated "threepeak" configuration, as shown in the "headers" above the panels of Figure $4 \mathrm{a}-\mathrm{e}$. Indeed, examples of such periodic chromatic variations in the stripe pattern are found experimentally, as reported in Figure S1, with various degrees of chromatic saturation.

This analysis demonstrates the impact of the mutual arrangements of defects on the spectral response of the films, even without involving the effect of pitch variation between domains.

Pitch Variation. Another simple way to create a defect in a cholesteric structure is to introduce different pitches throughout the sample thickness. This case is also a very important example, as pitch variations have been frequently reported in the literature. ${ }^{6,8,31}$ Based on our initial investigation, the effect of pitch variation between two regions is expected to also depend on the relative thickness of the two domains, and (dis)continuity of the director $\mathbf{n}$ at the interface, expressed by the relative angle $\varphi$. To investigate these various effects in a single model system, we simulated a progressive transition from a region of small to high pitch in a film of fixed total thickness $(2000 \mathrm{~nm})$ by splitting it at an oblique plane. In both cases, a uniform domain of pitch $p_{2}=340 \mathrm{~nm}$ at the far left is progressively replaced from below by a uniform domain of larger pitch $p_{3}=400 \mathrm{~nm}$, until it turns fully monodomain again at the far right.

Figure 4, column 4 (d, i, and $n)$, reports the first case, where there is no discontinuity in the director $\mathbf{n}$ at the junction $(\varphi=$ 0 everywhere). In the second case, reported in Figure 4, column 5 (e, j, and o ), the domains are independently oriented at the top and bottom interfaces and present a periodic mismatch at their junction $\left(\varphi=-q_{2} z=\left|q_{2}\right| z\right.$, where $\left.q_{2}=-2 \pi\right)$ $p_{2}$, with the negative sign chosen to account for the lefthandedness of the helicoid structure, and $z=h(1-r / L)$, where $h$ is the total sample thickness and $L$ is an arbitrary distance over which the two domains overlap with one another).

In the first case $(\varphi=0)$, the peak redshifts smoothly from one domain to the other, while in the second case (varying $\varphi$ ), clear oscillations are observed due to the periodic director mismatch. These observations clearly indicate that the periodic mismatch of the director rotation is the primary origin of these oscillations and strongly suggest that the structure was formed by the merger of independently formed cholesteric domains leading to a mismatching cholesteric order at the grain boundary.

It is important to consider that, while we defined the rotation angle $\varphi$ as the mismatch of the nematic director $\mathbf{n}$ at the interface of two defect-free sections, having an abrupt interface itself is not the cause of peak shifts. The real significance of $\varphi$ is the relative rotation of the bulk helices rather than the local discontinuity in the director field at their junction. This was verified by checking that small continuous twists introduced at the interface (to ensure a smooth transition of the director $\mathbf{n}$ ) led to only minor changes in the reflection spectrum.

Tilted Domains. The cholesteric domains can also be tilted away from the vertical direction, with a helical orientation described by a polar angle $\beta$ in the $(\mathbf{r}, \mathbf{z})$ plane and an azimuthal angle $\chi$ describing the orientation of $\mathbf{r}$ within the $(\mathbf{x}$, y) plane. As shown in Figure 5, a tilt of the domain can result in a periodic rotation of $\mathbf{n}$ in the $(\mathbf{x}, \mathbf{y})$ plane, along the scanning direction $\mathbf{r}$. This rotation is likely to lead to cyclic variations of $\varphi$ (as well as $\varphi^{\prime}$ ). Therefore, the stripy colored pattern observed in experimental conditions would then likely correspond to a tilted tactoid vertically compressed and sandwiched between two vertically aligned cholesteric domains.

The tilted nature of the domain can impose constraints on the observation of the defects, which motivates a couple of approximations considered here. First, for small enough tilts $\beta$, the optical response measured in reflection is very similar to a nontilted domain of similar pitch. The numerical aperture of the objective used in this work $(\mathrm{NA}=0.4)$ corresponds to a cone of incident and collection light of an angle of $\sin ^{-1}$ (NA) $\approx 23.6^{\circ}$, which sets an upper limit on $\beta$ for the tilted helices reflecting back into the objective as $\beta_{\max }=\frac{1}{2} \sin ^{-1}(\mathrm{NA} / n) \approx 7.5^{\circ}$ (n.b.: The fiber coupling to the spectrometer is limited to NA $=0.24$, leading to $\beta_{\max } \approx 4.4^{\circ}$, as described later). The mismatch between the pitch $p$ along the helical axis and its vertical component, $p_{z}$, is then $<1 \%$ and can therefore be neglected. Second, the optical response of a cholesteric structure under sufficiently small incidence angle can be at first approximated to the one at normal incidence.

The specificity of the self-assembly route, involving a vertical compression in the last stage of the drying process, causes an anisotropic distortion of the kinetically arrested cholesteric structure that affects both the pitch and the helical axis. The vertical compression during this stage causes varying changes in pitch depending on the helix tilt, with vertically aligned domains experiencing the greatest shrinkage. ${ }^{8}$ The variation in helix tilt expands the range of possible optical responses from a stripy defect compared to an ordered monodomain film. To investigate the complexity of the physical structure and guide further simulations, we performed direct observations using cross-sectional SEM.

Structural Analysis of a Defect with Stripy Pattern. Direct observation of the local structure within a stripy colored defect offers a better understanding of the different structural changes responsible for the resulting optical response. To this end, the hyperspectral imaging analysis was combined with an SEM cross-sectional analysis of the same defect, according to a previously developed procedure, and is illustrated in Figure $6 .{ }^{6}$ In short, we first fractured the film and isolated a domain where the crack was perpendicular to the stripy pattern. The film edge showing a stripy pattern was then optically analyzed 
a

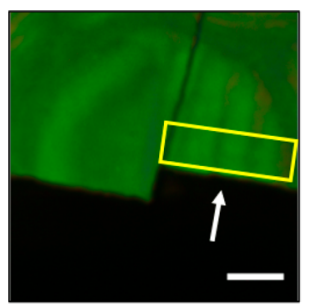

C
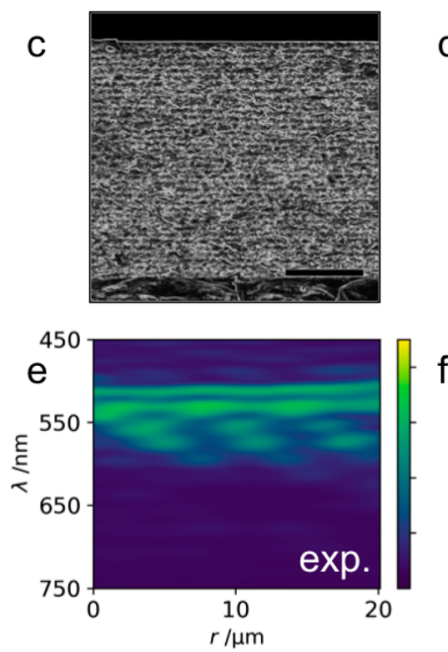

b

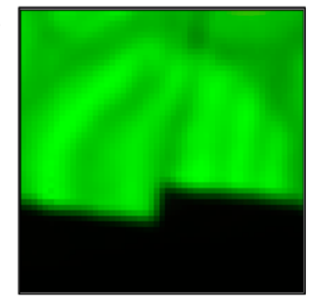

d

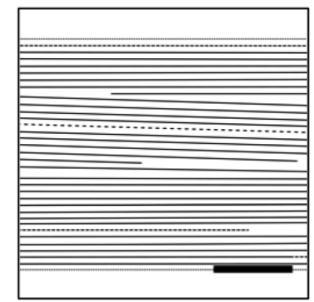

$f$

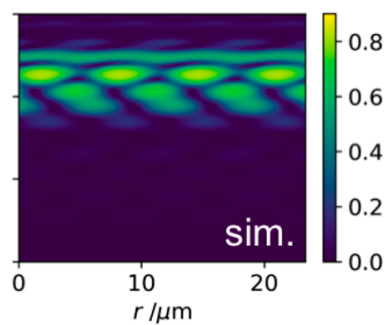

Figure 6. Direct comparison of experimental and simulated spectra of a defect. (a) Polarized optical microscopy illustrating the topview of a defect near a sharp section (scale bar $10 \mu \mathrm{m}$ ). (b) RGB image reconstructed from a hyperspectral scan of the same area. (c) SEM image (after processing) taken in cross-section in the region indicated in (a) by the arrow (scale bar $2 \mu \mathrm{m}$ ). Original image in Figure S7. (d) Schematic of the corresponding periodicities revealed from (c), revealing a middle domain tilted by $\beta \approx 1.8^{\circ}$ (dotted lines are plotted where original lines could not be seen). (e) Experimental hyperspectral scan of the defect, measured in reflection in the area marked by the yellow rectangle in (a). (f) Simulated hyperspectral scan generated using the domain thicknesses and pitches extracted from SEM analysis (more details in Figure S8).

in top view using both high-resolution polarization-resolved optical microscopy (Figure 6a) and hyperspectral analysis, from which an RGB image was reconstructed (Figure $6 \mathrm{~b}$ ). The region of interest was then marked with a razor to facilitate its relocation under SEM, allowing us to image the cross-section of the same region (Figure 6c, full range in Supporting Information, Figure S7) and to image the periodic arrangement of CNCs at the cleaved surface (Figure 6d). This analysis revealed a vertical stack with three sections. The top and bottom sections appear as horizontal lines parallel to the film edges, indicating vertically aligned domains $(\mathbf{m} / / \mathbf{z})$, in agreement with the expected effect of anchoring at both the liquid-substrate and the liquid-air interfaces. The middle section, on the other hand, appears to be tilted by $\beta \approx 1.8^{\circ}$ (to the right, i.e., clockwise in the $(\mathbf{r}, \mathbf{z})$ plane). Note that since the optical characterization was performed along an axis perpendicular to the cross-section, the azimuthal orientation of the helical axis away from the cross-sectional plane is negligible $\left(\chi \approx 0^{\circ}\right)$, and the apparent pitch, measured by the lines periodicities in the cross-section, is almost equal to the real pitch $\left(p_{\text {app }} \approx p\right)$, and the same is true for the apparent tilt $\left(\beta_{\mathrm{app}} \approx \beta\right) .{ }^{8,25}$ The measurement of apparent periodicities indicated, respectively, from top to bottom in the three different domains, pitch values $p_{1}=344 \mathrm{~nm}, p_{2}=350 \mathrm{~nm}$, and $p_{3}=330 \mathrm{~nm}$ and thicknesses $t_{1}=1.36 \mu \mathrm{m}, t_{2}=2.1 \mu \mathrm{m}$, and $t_{3}=$ $2.28 \mu \mathrm{m}$.

The cross-sectional analysis of the stripy defect from SEM was used to simulate a spectral signature that can be directly compared to the experimental hyperspectral signature analysis of the film recorded in the top view. However, the exact relative orientation between the director field in the top and bottom domains, which corresponds to the term $\varphi^{\prime}+\varphi$, is difficult to estimate from the SEM image and hence was left as a free, adjustable parameter. Figure 6 f shows that using $\varphi^{\prime}+\varphi$ $=77^{\circ}$, we produced a simulated spectral signature of the stripy pattern in excellent agreement with the experimentally measured one. Note that shearing of the structure during drying results in a distorted helicoidal order along its effective helical axis $\mathbf{m}$. ${ }^{8}$ The optical index oscillation between $n_{\mathrm{e}}$ and $n_{\mathrm{o}}$ along the helical axis therefore deviates from being purely sinusoidal. We found that this effect appeared negligible for such a low tilt $\beta \approx 2^{\circ}$ (distortion $\sim 6 \%$, see Figure S8 for details). In the simulated spectra, only normal incidence was considered for simplicity, since the cone of light for illumination and collection turned out to be significantly smaller than expected from the objective numerical aperture $(\mathrm{NA}=0.4)$, due to the smaller NA for the optical fiber coupling into the microscope (measured as NA $=0.24$ in the K-space). Indeed, an effective NA of 0.4 would not allow for the observation of the spectral fringes in Figure 6e (spaced by $\sim 20 \mathrm{~nm}$ ). From combining Bragg's and Snell's laws, the maximal possible shift in the reflected spectra is $\lambda_{\mathrm{n} . \mathrm{i}}-\lambda=\lambda_{\mathrm{n} . \mathrm{i} .}\left(1-\sqrt{1-(\mathrm{NA} / n)^{2}}\right) \approx 6.6 \mathrm{~nm}$ and 18.5 $\mathrm{nm}$, for NA $=0.24$ and 0.4 , respectively, for a peak centered at normal incidence at $\lambda_{\mathrm{n} . \mathrm{i}}=550 \mathrm{~nm}$.

The transfer matrix method has a number of limitations, mostly notable the requirement that the medium is stratified into parallel layers, leading to inaccuracies in simulating offparallel domains, and the assumption that the light source is fully coherent. Transfer matrix calculations therefore fall short when simulating small defects and thick films. Despite these limitations, the excellent agreement seen in Figure 6 demonstrates the potential that hyperspectral imaging offers to understand the formation and local structure of multidomain cholesterics.

Mapping of the Helical Tilt at the Micron Range. The asymmetry of the spectral oscillations reported in Figure 2 are characterized by progressive blue-shifts alternating with abrupt red-shifts as we scan along one direction of the stripes. To understand this behavior, we defined the direction indicated by the arrows as the direction of increasing $r$. As shown above, this asymmetry arises from the dependence of the $\varphi(r)$ angle at the boundary between the tilted and the vertically aligned domain immediately above or below it. The sequential order of these two domains (the vertical domain being above or below) determines whether we are actually considering $\varphi(r)$ or $\varphi^{\prime}(r)$, which have opposite slopes with respect to $r$. As the direction of the tilt (i.e., which side of the stripes the domain is tilted) reverses the sign of $r$, we investigated the angular response of specific defects in the reciprocal space (K-space imaging) to univoquely determine the tilting direction.

Lifting Uncertainty of the Helical Axis Azimuthal Orientation. Due to the symmetry of the cholesteric order, the helical axis direction $\mathbf{m}$ can be arbitrarily defined either way parallel to that axis. By adopting the convention of $\mathbf{m}$ pointing 

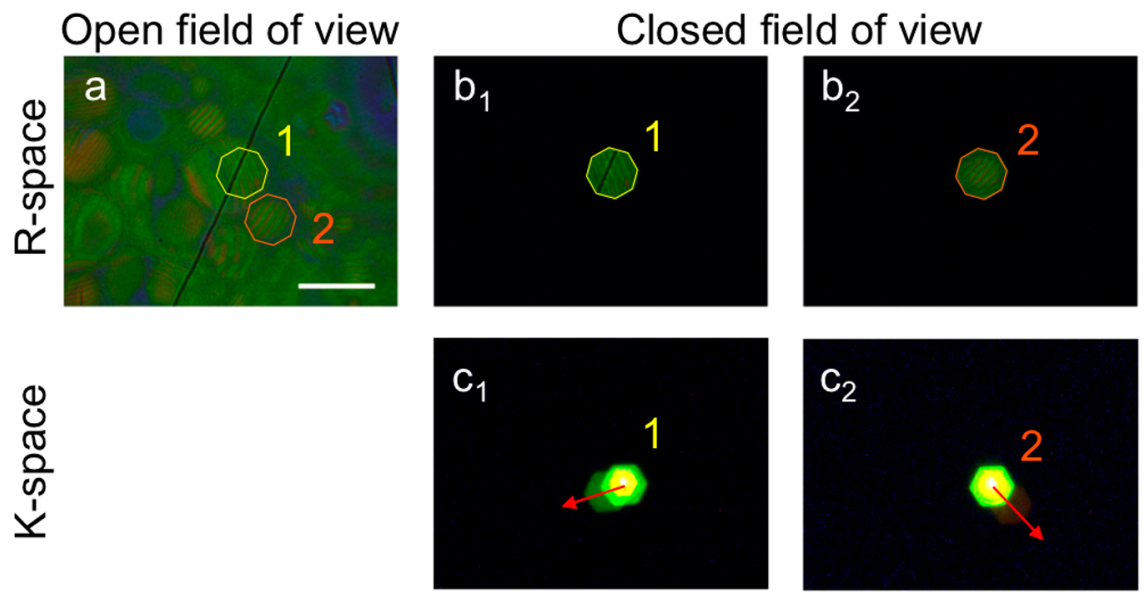

Figure 7. Determination of the orientation of isolated domains using spatially resolved K-space imaging. (a) Optical microscopy image (bright field, in reflection) illustrating two defects, labeled as 1 (from Figure 1) and 2. (b) The incident light is restricted to the field of view of each of these domains $\left(b_{1}\right.$ and $b_{2}$, respectively, area $\left.\sim 30 \times 30 \mu \mathrm{m}^{2}\right)$. (c) K-space imaging of the light reflected specifically by these two domains $\left(c_{1}\right.$ and $c_{2}$, respectively), in agreement with direction and expected fringe asymmetry (n.b.: The black line running through the domain 1 is a crack that appeared later in the film).
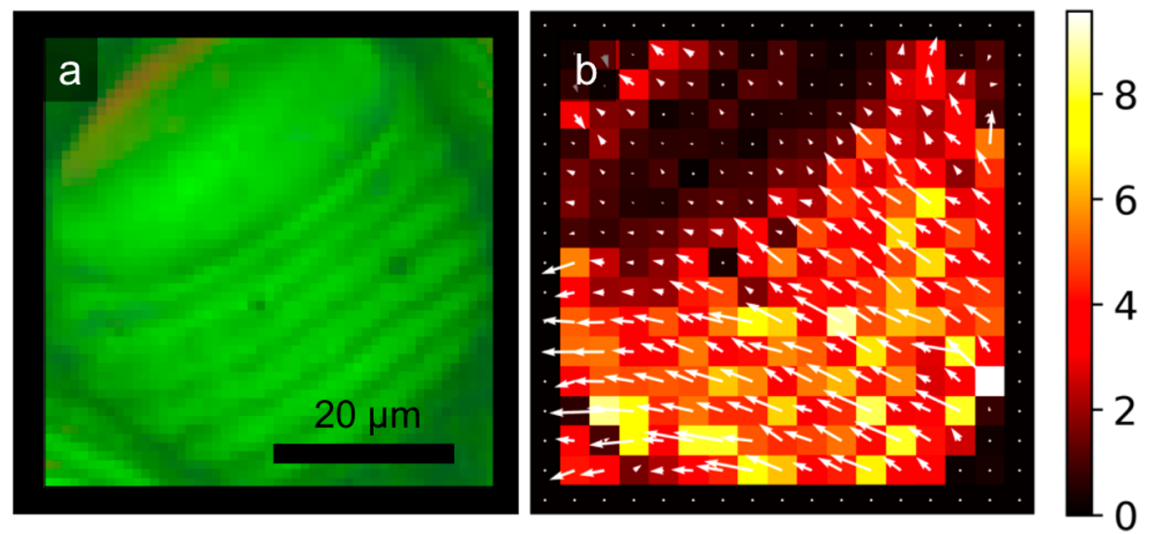

Figure 8. Extraction of local tilt from the hyperspectral analysis. (a) RGB reconstruction from hyperspectral scan of the defect 1 and (b) corresponding top view mapping of its helical axis orientation (azimuthal orientation given by white arrows, polar tilt in degrees expressed as an intensity heatmap), as deduced from the hyperspectral analysis, at a resolution of $3 \times 3 \mu \mathrm{m}^{2}$.

upward, we allow for the non-ambiguous determination of its azimuthal angle within $\left[0^{\circ}, 360^{\circ}\right]$. Since the direction of the stripy pattern is $180^{\circ}$-periodic, the uncertainty of the orientation of the helical axis, defining the direction of $r$ axis and therefore of $\varphi(r)$, was lifted using spatially resolved $\mathrm{K}$ space imaging of isolated domains (Figure 7). Since the reflected spot of light follows the direction of the helical axis, we found that the azimuthal projection of $\mathbf{m}$, parallel to $+\mathrm{z} \times$ $(\mathbf{m} \times \mathbf{z})$, is oriented against the scanning direction in Figure 2 and matches with the direction of progressive red-shift. This means that the phase shift contributing most in the defect hyperspectral oscillations increases with $\pi r / \Delta$, matching with the definition of $\varphi(r)$ and not $\varphi^{\prime}(r)$, which is indicative that the tilted domain is below the horizontal one.

Mapping the Helical Axis with Subdomain Resolution. The in-plane projection of the helical axis can be extracted from the asymmetry of the hyperspectral signature, allowing an easy mapping of its orientation with a spatial resolution of few microns. To demonstrate this, we performed such mapping on the same defect observed in Figure 2, using a pixel resolution of $3 \times 3 \mu \mathrm{m}^{2}$ (Figure 8). Such mapping reveals local distortions of the helical axis in azimuthal directions, while abrupt variations of the in-plane magnitude are also observed.
This fine mapping of the structure provides access to the spatial distortion of the helical field $\mathbf{m}$, often used as a coarsegrained description of the cholesteric director field $\mathbf{n}$. Such fine mapping at micron-range resolution can be exploited to isolate additional effects at play at these length scales.

From a Self-Assembly Model to a Reference Chart of Defects. In light of these observations, it is interesting to discuss the CNC self-assembly model upon drying. We observed that the top and the bottom domains are both aligned with the interface, while the tilted regions are entrapped in the middle. Sedimentation of tactoids and planar anchoring favors a vertical alignment of the cholesteric helix in the bottom domains, but this scenario cannot explain satisfactorily the vertical alignment of the top domains, as we will develop further. The top and bottom domains also present slightly different pitch values, with a smaller pitch in the bottom phase (top: $p_{1}=344 \mathrm{~nm}$, bottom: $p_{3}=330 \mathrm{~nm}$ ). This feature, which is not accounted for in our previous model, ${ }^{8}$ suggests a different self-assembly history for the top and bottom regions, with possible fractionation of CNCs taking place. ${ }^{14,20,32}$ Second, the periodicity $\Delta \approx 6.6 \mu \mathrm{m}$ and the pitch of the central domain $p \approx 350 \mathrm{~nm}$ suggest a tilt $\beta \approx 2.2^{\circ}$, in fair agreement with the estimations from $\operatorname{SEM}\left(\beta \approx 1.8^{\circ}\right)$. Third, 

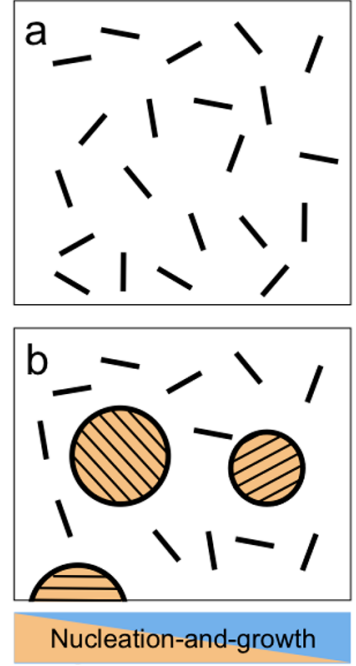

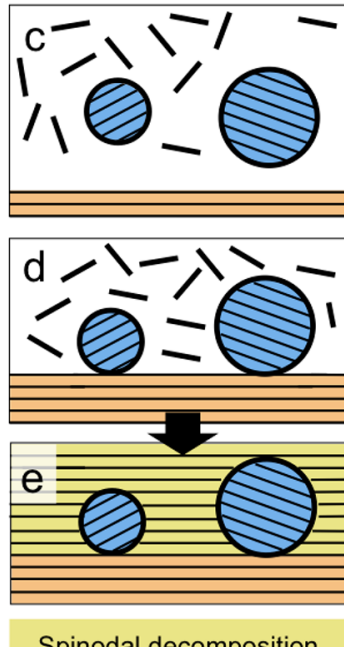

Spinodal decomposition

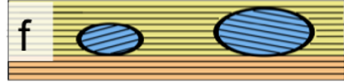

g

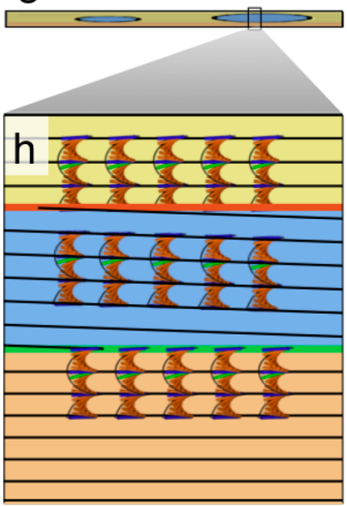

Figure 9. Schematic of the proposed CNC self-assembly pathway upon solvent evaporation, illustrated in cross-section. (a) Isotropic suspension, (b) NG via tactoid formation and sedimentation, (c) planar anchoring at the bottom interface, (d) remaining tactoids trapped at random orientation and vertical compression of the isotropic phase, favoring horizontal CNC alignment, (e) after spinodal decomposition (SD), black arrow between (d) and (e), the compressed isotropic phase turned into a vertically aligned cholesteric domain (n.b.: The top region, represented in yellow, is mostly aligned vertically but might be locally disturbed by the tactoid obstacles), (f) vertical compression after kinetic arrest, $(\mathrm{g})$ final film with defects reminiscent of the entrapped tactoids, and (h) magnified view showing a tilted middle domain and the vertically aligned top and bottom domains.

the compression model predicts that a tilted domain is expected to red-shift as the tilt $\theta$ increases and its associated periodicity $\Delta$ is expected to decrease. This latter observation is qualitatively confirmed in Figure S1, as the stripy defect patterns are consistently red-shifted by various amounts with respect to the large blue regions. Moreover, these blue regions present circular concentric defects with the largest associated periodicity $\Delta$. From the analogy with the previously described stripy colored patterns, we interpret these concentric defects as resulting from vertically aligned spherical tactoids sharing a flattened, dome-shaped boundary with domains immediately above them.

The self-assembly of CNCs from liquid suspension to solid film relies on several global parameters (vertical compression ratio, pitch at kinetic arrest, total film thickness, optical indices of cellulose, etc.) and local parameters (orientation of a tilted domain, size of the initial tactoid). This should lead, for a given CNC film, to a family of possible defects that all share the same constraints, as they belong to the same film. From our understanding of the self-assembly and the optical response of the defects, we can easily build a family of defect patterns that we can expect to encounter in our film and compare it to the details displayed in Figure $1 b, c$ and Figure S1. Once a calibration chart that satisfactory reproduces a family of possible patterns is constructed, it is possible to infer the local parameters that controlled any defect of interest. Examples of such charts are provided in Figures S9 and S10. This chart will be specific to the suspension used, film thickness, etc. and allows for a better understanding of the self-assembly processes. The specificity of defect patterns in CNC films could be harnessed for security printing based on defect recognition, with each thin film offering a nonreproducible set of defects possessing a hierarchy of details from millimeter down to the micron scale.

Comparison with Angular-Resolved Optical Spectroscopy. The analysis of individual defects could produce results lacking statistical significance. We therefore performed complementary analysis using angular-resolved optical spectroscopy, a technique successfully used in previous studies for these systems and easily applied to larger sample areas (typically from ca. $1-30 \mathrm{~mm}^{2}$ ). ${ }^{8,25}$ A scattering scan was obtained by initially fixing the incident angle $\theta_{\mathrm{i}}$ and outgoing angle $\theta_{\mathrm{o}}$ both equal to $\gamma=45^{\circ}$ (i.e., in specular conditions) and then exploring the optical response as the sample was tilted by an angle $\hat{\phi}$, while the sum $\theta_{\mathrm{o}}+\theta_{\mathrm{i}}=2 \gamma$ was kept constant (more details in Supporting Information). The angular scattering signature (Figure S11) reveals a wavelength dependence $\lambda(\hat{\phi})$ and thus $p(\beta)$, suggesting $\alpha \approx 0.101 \pm 0.004$ and $p_{\mathrm{ka}} \approx 3450 \pm$ $50 \mathrm{~nm}$. These values, which are taken from a larger area and are therefore more statistically robust, are in good agreement with those estimated from the analysis of the defect from Figure $6\left(\alpha \approx 0.1, p_{\mathrm{ka}} \approx 3300 \mathrm{~nm}\right)$, although the defects in Figures 2 and 6 correspond to a different area of the film, which could cause some variations. Moreover, scanning the illumination angle $\theta_{\mathrm{i}}$ while maintaining $\theta_{\mathrm{o}}=\theta_{\mathrm{i}}$ allowed for the characterization of the specular response, indicative of the wellaligned domains corresponding to the top and bottom regions (Figure S12). This scan reveals an asymmetric peak (in intensity $v s$ wavelength), suggesting the overlapping of a main peak and a secondary peak at a slightly longer wavelength. This is in qualitative agreement with the presence of a more reflective bottom domain (being also thicker, $t_{3}>t_{1}$ ) overlapping with the existence of a thinner domain of a slightly larger pitch $\left(p_{1}>p_{3}\right)$. Figure S12 also reveals additional bands both at shorter and longer wavelengths with respect to the main specular peak, which we ascribe to secondary peaks analogous to those explained in Figure S5. While angularresolved optical spectroscopy allows us to refine the statistical description of the film and confirm some observed trends observed from HSI, it is insufficient on its own to correctly interpret the complexity of the optical response as resulting from a layered structure caused by the defects.

Evolution from Nucleation and Growth to Spinodal Decomposition. The numerical reproduction of the exper- 
imental features observed in the defects allows us to understand the possible phase transition mechanisms responsible for this pattern formation. The presence of these striped circles, reminiscent of the entrapped cholesteric tactoids, represents another confirmation, among many others, ${ }^{2,14,20,32,33}$ of the role of NG as the driving phase transition mechanism from isotropic to cholesteric in $\mathrm{CNC}$ suspensions upon casting. Nevertheless, it is clear from SEM cross-section, optical microscopy, and HSI analysis that the medium immediately above these trapped tactoids is also cholesteric. This observation ultimately poses the question of the driving mechanism of the phase transition of this top domain into a cholesteric order. The sharp defect interface between the trapped tactoids and the top domain invalidates the possibility that the cholesteric order propagated from these tactoids to the CNCs in the top domain and suggests the tactoids did not grow further. For that reason, NG alone is not enough to explain this pattern formation. Another mechanism must be responsible for the transition of the top domain into the cholesteric order, namely SD. The onset of such a transition between the two processes has been experimentally predicted and reported in colloidal suspensions of rigid rods and is indeed expected to depend on the volume fraction at which they were quenched: favoring NG at lower volume fraction and SD at higher. ${ }^{34,35}$ A schematic summarizing the proposed self-assembly route is proposed in Figure 9. To our knowledge, only one work reported this self-assembly pathway in suspensions of very long CNCs (few microns) extracted from tunicate. ${ }^{36}$

An origin of such a quench can be found in the casting conditions: The solvent evaporation occurring from the liquid-air interface causes the sample volume to decrease rather linearly with time, and thus the $\mathrm{CNC}$ volume fraction increases hyperbolically. As a result, the volume fraction increases much faster in the latest stages of the assembly, when the CNCs located above the trapped tactoids are forced to pack in a much shorter amount of time to form a cholesteric phase. NG requires an induction time, while SD does not. This induction time arises from the metastability of the isotropic phase within the binodal regime, when the top CNC suspension becomes supersaturated, and an activation energy is required to nucleate tactoids. Note that in colloidal systems, as compared to in molecular systems, impurities are less likely to act as nucleation points, so the induction time remains clearly observed. ${ }^{37}$ The higher rate at which the volume fraction increases upon drying is thus likely to increase the supersaturation of the top CNC suspension beyond the metastable regime, before the top CNC suspension has the opportunity to undergo a NG process. The required induction time for NG is also increased by the increasing hydrodynamic interactions between CNCs as the volume fraction increases. All these factors can contribute to make CNCs too slow to assemble into tactoids and can trigger a second regime where the isotropic phase is thermodynamically unstable, the activation energy vanishes, and any local fluctuation of CNC orientation causes SD to start immediately.

The role of polydispersity and local interactions between CNCs (either repulsive or attractive, and evolving as the ionic strength increases upon drying) are likely to modify how the CNCs phase separate and interact. CNCs are known to be highly polydisperse in length, and fractionation effects have been observed in biphasic suspensions. ${ }^{38}$ Interestingly, polydispersity in suspensions of hard rods has been reported not only to extend the metastable volume fraction range but also to bring the volume fraction threshold for SD very close to the threshold where the isotropic phase becomes unstable. ${ }^{34}$ The latter fact also strongly supports that the CNCs in the top domain underwent a SD process as a result of a concentration quench upon drying. The fractionation effect occurring in the biphasic regime causes the longer $\mathrm{CNCs}$ to assemble first into a cholesteric phase, while the shorter CNCs accumulate in the isotropic phase. ${ }^{2,38-40}$ If this fractionation occurred also in situ in the cast suspension upon drying, the volume fraction of $\mathrm{CNC}$ remaining in the isotropic phase (that became the top domain) can be significantly higher than the one at which the tactoids appeared (easily by a factor $\sim 2$ ). This higher volume fraction not only increases the hydrodynamic interactions between CNCs (slowing down the motions of individual CNCs; and ionic strength increase could also amplify it) but also postpones the phase transition to a more advanced drying stage when the rate of volume fraction increase is much higher (for a factor 2 in volume fraction, we can expect a factor 4 in the concentration rate). Finally, Monte Carlo simulations on monodisperse rods of shorter aspect ratios showed inhibition of NG and promotion of SD followed by dynamic arrest, which could imply a similar scenario after accumulation of shorter CNCs in the isotropic phase. ${ }^{41}$ For these reasons, the dimensions of the CNCs, and also their polydispersity and the suspension rheological properties, are likely to impact the chosen self-assembly pathway. ${ }^{38,42}$

The relative homogeneity of the top cholesteric domain might at first appear to be in conflict with a SD scenario, since this scenario usually leads to characteristic patterns of fluctuations of volume fraction that we do not observe here but that are otherwise widely reported in typical experiments. Several important differences have to be mentioned to understand why we actually do not expect these fluctuations here. First, SD is known to develop characteristic patterns of fluctuations of volume fraction that evolve in time toward a coarse-grained structure of low and high densities, which eventually yield a macrophase separation after full equilibration (i.e., if no kinetic arrest hinders it). Most studies focus on triggering them with well-controlled quenches, followed by careful observations and measurements of their characteristics over time without disturbing their evolution. Typical observations reveal that these fluctuations are initially continuous, and no sharp interface exists between the ordered, high density phase and the isotropic, disordered phase in coexistence. ${ }^{34}$ If the imposed concentration quench is shallow (which is expected here since the volume fraction increases continuously), the developed fluctuations are expected to be of longer range. ${ }^{43}$ For the remaining isotropic CNC suspensions that undergoes upon drying a SD process, the continuously increasing volume fraction acts as a continuously quenching condition that does not allow the isotropic, lower density regions to develop, while the aligned, higher density regions propagate and fill the space. In that condition, no sharp interface is expected to survive this process once the coexisting isotropic phase has undergone transition into a full cholesteric.

A final and key phenomenon to explain the absence of patterns, as well as the perfect vertical alignment of the CNCs in the top CNC domain, is the anisotropy of the suspension compression causing the concentration quench. The solvent (here water) evaporated from the liquid-air interface, while the lateral dimensions of the drying suspensions are constrained by pinning on the dish edges. This causes a vertical 
evaporation front, propagating downward, that eventually breaks the macroscopic symmetry of the supersaturated isotropic phase. In this condition, the $\mathrm{CNCs}$ are on average oriented away from the vertical direction, which in consequence selects the vertical direction for the development of the helical axis of the appearing cholesteric phase. Since this symmetry breaking is uniform throughout the cast suspension, the vertical direction for the cholesteric order develops homogeneously throughout the top domain, allowing no recognizable pattern to develop, and reaches out even into the interstices between the entrapped tactoids. Importantly, the alternative conception that anchoring from the top interface is responsible for the alignment of the top domains does not hold, since propagation of the vertical alignment from the interface to these interstices would have been overcome by a different alignment propagating from the tactoids themselves (note that the anchoring scenario might also imply a local concentration gradient near the top interface, which is hydrostatically unstable due to its higher density compared to the isotropic phase, making it unfavorable). The macroscopic symmetry-breaking condition to quench the top CNC suspension at a higher volume fraction also favors kinetically the evolution toward this uniform cholesteric domain: Instead of relying on local, spontaneous fluctuations of CNC orientation of conflicting orientations that then poorly coordinate their mutual alignment, here the uniform vertical compression experienced by the CNCs certainly enhances the kinetics of the cholesteric formation and their uniformity on a larger scale.

This unusual scenario shares some analogy with a system of prealigned rods, where the alignment is maintained in the SD process. ${ }^{44}$ While the macroscopic alignment is expected to hold on average, it suggests that the possibility of transient vacuoles (that would eventually collapse upon drying) and presence of tactoids (as obstacles around which the isotropic phase is being compressed) can affect the quality of the local alignment.

The existence of these two alternative self-assembly pathways on the formation of photonic CNC films necessarily raises the question of their individual control, from the choice of CNCs to the suspension conditions. A more in-depth understanding would, however, require a dedicated study of these mechanisms as they occur in suspension or, even better, in situ upon solvent evaporation. This could involve monitoring the suspension in glass cuvettes to allow for in situ optical microscopy and laser diffraction upon solvent evaporation, ${ }^{45,46}$ while more conventional post-shear relaxation could be performed on isolated isotropic phases fractionated at various stages to locate the concentration region of spinodal instability. ${ }^{33,43,47}$ Finally, clarifying the interplay between SD and kinetic arrest, via optorheological studies, ${ }^{48,49}$ would also be insightful, as suggested by recent observations on CNC suspensions at higher ionic strength. ${ }^{38,50}$

\section{CONCLUSIONS}

In conclusion, this work demonstrates the promising potential of hyperspectral imaging to extract the local optical response of CNC films in a fast and nondestructive manner. This powerful experimental tool can locally resolve various spectral signatures in a sample and discriminate overlapping contributions from, for example, structural architecture or the presence of chiral or achiral guest molecules. It also complements other optical techniques, such as large-area angular-resolved optical spec- troscopy, for the systematic investigation of the self-assembled photonic structures found in CNC films. Our technique was validated by comparing experimental hyperspectral cross sections to the simulated responses obtained from crosssectional SEM along a "stripy colored pattern", revealing existence of superposed, distorted cholesteric domains of various pitch and tilts, reminiscent of tilted tactoids embedded into a cholesteric continuum, as further supported by spatially resolved K-space imaging. The obtained observation allowed the verification of the CNC compression model on a subdomain level. ${ }^{8}$

The derived defect structure strongly supports a temporal evolution, occurring upon solvent evaporation, of the phase transition from nucleation and growth (NG, for the defects reminiscent of tactoids) to spinodal decomposition (SD, for the top domain immediately above them). The implications of this alternative mechanism on the self-assembly of CNC films, in terms of uniformity, optical response, etc., can be farreaching and explain some so far unexplained differences between the overall optical "quality" of CNC films between different manufacturers, extraction procedures, formulations, and self-assembly protocols.

Most efforts to control the self-assembly of colloidal nanocrystals into ordered structures rely on strategies assuming NG mechanism, ${ }^{51}$ whereas SD is employed to generate more disordered patterns. ${ }^{52}$ This work strongly suggests that SD can also result in well-ordered structures. In this particular case, this ordered outcome arose from the lyotropic behavior of anisometric particles, where the kinetic nature of the SD pathway is driven by orientational rather than positional fluctuations, combined with the pre-existing alignment induced here by the unidirectional contraction of the sample upon drying. These conditions should be relevant to a broader range of colloidal particles assembled in dynamically evolving conditions where volume is not conserved.

\section{EXPERIMENTAL METHODS}

Materials. The initial suspension of $14.5 \mathrm{wt} \%$ cellulose nanocrystals (CNCs) was prepared from filter paper, as described below, with subsequent formulations diluted with deionized water (Millipore Milli-Q gradient A10, resistivity $>18 \mathrm{M} \Omega \cdot \mathrm{cm}^{-1}$ ).

Cellulose Nanocrystal Preparation. CNCs were obtained from the hydrolysis of Whatman no. 1 cellulose filter paper $(30 \mathrm{~g})$ with sulfuric acid $\left(64 \mathrm{wt} \%, 420 \mathrm{~mL}\right.$ ) at $64{ }^{\circ} \mathrm{C}$ for $30 \mathrm{~min}$, before being quenched using milli-Q ice and water. ${ }^{19}$ Soluble cellulose residues and acid were removed by centrifugation (three steps at 20,000 $\mathrm{g}$ for 20 $\mathrm{min}$ ) and dialysis against deionized water (MWCO 12-14 $\mathrm{kDa}$ membrane), and a stable suspension of $[\mathrm{CNC}]=2.21 \mathrm{wt} \%$ was obtained. Conductivity titration against sodium hydroxide indicated $\left[-\mathrm{OSO}_{3}^{-}\right]=205 \mathrm{mmol} \cdot \mathrm{kg}^{-1}$ of $\mathrm{CNC}$. The suspension was tipsonicated in an ice bath (Fisherbrand Ultrasonic disintegrator $500 \mathrm{~W}$, amplitude $30 \%$ max, duration $48 \mathrm{~s}$, suspension volume $40 \mathrm{~mL}$ ) and vacuum-filtered ( $8.0 \mu \mathrm{m}$ then $0.8 \mu \mathrm{m}$ nitrocellulose, Sigma-Aldrich). The suspension was partially desulfated and concentrated by heating at $60^{\circ} \mathrm{C}$ in a water bath for $12 \mathrm{~h}$ (lid on) and additional $3 \mathrm{~h}$ at $40^{\circ} \mathrm{C}$ (lid off), resulting in a $14.5 \mathrm{wt} \%(\sim 9.4 \% \mathrm{v} / \mathrm{v})$ suspension of CNCs. More information on this suspension (conductometric titration, ionic content, phase diagram, pitch vs concentration) can be found in ref 19.

Cellulose Film Preparation. Films were prepared by casting a dilute suspension of CNC ( $1 \mathrm{~mL}, 1.5 \mathrm{wt} \%)$ on a polystyrene Petri dish of $3.5 \mathrm{~cm}$ diameter and allowing it to dry in ambient conditions. In order to slow down the evaporation, the dish was covered with parafilm in which many small holes were punctured (drying time estimated to about 5 days). 
Optical Microscopy and Hyperspectral Imaging. Optical characterization was performed in reflection mode on a customized Zeiss Axio Scope.A1 modular microscope using a halogen lamp (Zeiss HAL100) as a light source with Koehler illumination and a PriorXY motorized stage with movements in $x, y$, and $z$ axes of submicron precision. The light reflected off the sample is split between a CCD camera (UI-3580LE-CHQ IDS) and an optical fiber (Avantes, Ø 50 $\mu \mathrm{m})$ mounted in confocal configuration and connected to a spectrometer (AvaSpec-HS2048XL, Avantes). A superlong working distance objective (Nikon SLWD T Plan) of high magnification (50X , $\mathrm{NA}=0.4, \mathrm{WD}=22 \mathrm{~mm}$, focal length $4 \mathrm{~mm}$, min physical depth of focus $1.72 \mu \mathrm{m}$ ) was used for automated measurements. Normal optical microscopy images were recorded directly from the camera. Hyperspectral imaging was performed using a script to command the PriorXY motorized stage for an automatized XY scanning of a specific area of the sample and acquire in each location the reflected spectra over a small sampling area coupled to the optical fiber to the spectrometer. With the $50 \times$ objective, the sampling area is a disk of $\varnothing$ $\sim 2 \mu \mathrm{m}$. Spline interpolation was used for spatial resolution in the plots of changing spectrum $v s$ displacement. A Savitzky-Golay filter was used to smooth spectral data. From the spectra, the CIE standards were used to produce equivalent RGB colors and reconstruct colored images. ${ }^{28}$ For area-specific K-space imaging, first the field aperture was closed around a region of interest, then a Bertrand lens was inserted in the back of the objective (after the carrousel, so that the incident light is not passing through it), and the aperture diaphragm was closed. The focal lens of the Bertrand lens was previously conjugated to the objective rear focal plane.

Angular-Resolved Optical Spectroscopy. Measurements were carried out using a lab-made goniometer: A xenon lamp (HPX-2000, Ocean Optics) was used as the light source, and a spectrometer (AvaSpec-HS2048XL, Avantes) was used to analyze the scattered optical signal. The sample was mounted on a rotating stage in the center of the goniometer and illuminated with a collimated incident beam focused with a $300 \mathrm{~mm}$ focus on the sample surface (light spot size $\varnothing \sim 1 \mathrm{~mm}$ ). A detector was mounted on an arm attached to a motorized rotation stage and coupled the scattered light into an optic fiber connected to the spectrometer. The recorded light intensity was normalized with respect to a white Lambertian diffuser, while the exposure time was adjusted using an automatized high dynamic range method. ${ }^{53}$ Measurements were recorded at a fixed angle $\gamma=90^{\circ}$ between incident and outgoing beams by scanning the sample tilt $\hat{\phi}$, defined from the angular bisector of the two beams (e.g., $\hat{\phi}=0^{\circ}$ corresponds to specular conditions).

Scanning Electron Microscopy Imaging. Scanning electron microscopy (SEM) images were acquired using a Zeiss Leo Gemini 1530VP system, working at $90^{\circ}$ with respect to the electron beam. SEM samples were mounted on aluminum stubs using conductive carbon tape and, to minimize surface charging, sputtered with a 5-10 $\mathrm{nm}$ layer of $\mathrm{Au} / \mathrm{Pd}$ (Emitech K550; I = 55 mA for $14 \mathrm{~s}$ ). The acceleration voltage used was $4.0 \mathrm{kV}$, and the working distance was $3.2 \mathrm{~mm}$.

Optical Calculations. The optical response of cholesteric structures was modeled using the transfer matrix method formalism based on Berreman's $4 \times 4$ matrix method. ${ }^{54,55}$ It was computed using a code package developed especially for this work (https://github. $\mathrm{com} /$ zhubonan/tm4) and made open source under a MIT license. Its design was inspired by an already existing Python implementation of the Berreman's $4 \times 4$ matrix method. ${ }^{56}$ The database of stripes (Figures S9 and S10) was computed using PyLlama (https://github. com/VignoliniLab/PyLlama), a Python toolkit implementing Berreman's $4 \times 4$ matrix method in a flexible way for any multilayer system. ${ }^{57}$

\section{ASSOCIATED CONTENT}

\section{(s) Supporting Information}

The Supporting Information is available free of charge at https://pubs.acs.org/doi/10.1021/acsnano.0c05785.
Numerical methods, higher resolution images, schematic of hyperspectral imaging setup, simulated spectra, crosssectional SEM, and reference charts (PDF)

\section{AUTHOR INFORMATION}

\section{Corresponding Authors}

Bruno Frka-Petesic - Department of Chemistry, University of Cambridge, Cambridge CB2 1EW, United Kingdom; ○ orcid.org/0000-0001-5002-5685; Email: bf284@ cam.ac.uk

Silvia Vignolini - Department of Chemistry, University of Cambridge, Cambridge CB2 1EW, United Kingdom; 다이.org/0000-0003-0664-1418; Email:sv319@ cam.ac.uk

\section{Authors}

Bonan Zhu - Department of Chemistry, University of Cambridge, Cambridge CB2 1EW, United Kingdom; (1) orcid.org/0000-0001-5601-6130

Villads E. Johansen - Department of Chemistry, University of Cambridge, Cambridge CB2 1EW, United Kingdom; (1) orcid.org/0000-0001-5921-7237

Gen Kamita - Department of Chemistry, University of Cambridge, Cambridge CB2 1EW, United Kingdom; (1) orcid.org/0000-0003-3923-7606

Giulia Guidetti - Department of Chemistry, University of Cambridge, Cambridge CB2 1EW, United Kingdom; (1) orcid.org/0000-0002-6065-3359

Mélanie M. Bay - Department of Chemistry, University of Cambridge, Cambridge CB2 1EW, United Kingdom; (1) orcid.org/0000-0001-8394-6712

Thomas G. Parton - Department of Chemistry, University of Cambridge, Cambridge CB2 1EW, United Kingdom; (1) orcid.org/0000-0001-7153-1042

Complete contact information is available at: https://pubs.acs.org/10.1021/acsnano.0c05785

\section{Notes}

The authors declare no competing financial interest. The raw data supporting this research are made freely available on the Cambridge data repository at this address: https://doi. org/10.17863/CAM.53665.

\section{ACKNOWLEDGMENTS}

This work was supported by the BBSRC [David Phillips fellowship BB/K014617/1], the EPSRC [1525292, EP/ R511675/1, EP/K503757/1, EP/L015978/1], the Isaac Newton Trust Cambridge [76933], the European Research Council [ERC-2014-STG H2020 639088, ERC-PoC-2017 790518] and the European Commission, Marie Skłodowska-Curie Action [LODIS 701455].

\section{REFERENCES}

(1) Lagerwall, J. P. F.; Schütz, C.; Salajkova, M.; Noh, J.; Hyun Park, J.; Scalia, G.; Bergström, L. Cellulose Nanocrystal-Based Materials: From Liquid Crystal Self-Assembly and Glass Formation to Multifunctional Thin Films. NPG Asia Mater. 2014, 6 (1), No. e80.

(2) Schütz, C.; Bruckner, J. R.; Honorato-Rios, C.; Tosheva, Z.; Anyfantakis, M.; Lagerwall, J. P. F. From Equilibrium Liquid Crystal Formation and Kinetic Arrest to Photonic Bandgap Films Using Suspensions of Cellulose Nanocrystals. Crystals 2020, 10 (3), 199. 
(3) Kontturi, E.; Laaksonen, P.; Linder, M. B.; Nonappa, A. H.; Gröschel, A. H.; Rojas, O. J.; Ikkala, O. Advanced Materials through Assembly of Nanocelluloses. Adv. Mater. 2018, 30 (24), 1703779.

(4) Habibi, Y.; Lucia, L. A.; Rojas, O. J. Cellulose Nanocrystals: Chemistry, Self-Assembly, and Applications. Chem. Rev. 2010, 110 (6), 3479-3500.

(5) Parker, R. M.; Guidetti, G.; Williams, C. A.; Zhao, T.; Narkevicius, A.; Vignolini, S.; Frka-Petesic, B. The Self-Assembly of Cellulose Nanocrystals: Hierarchical Design of Visual Appearance. Adv. Mater. 2018, 30 (19), 1704477.

(6) Dumanli, A. G.; van der Kooij, H. M.; Kamita, G.; Reisner, E.; Baumberg, J. J.; Steiner, U.; Vignolini, S. Digital Color in Cellulose Nanocrystal Films. ACS Appl. Mater. Interfaces 2014, 6 (15), 1230212306.

(7) Frka-Petesic, B.; Guidetti, G.; Kamita, G.; Vignolini, S. Controlling the Photonic Properties of Cholesteric Cellulose Nanocrystal Films with Magnets. Adv. Mater. 2017, 29 (32), 1701469.

(8) Frka-Petesic, B.; Kamita, G.; Guidetti, G.; Vignolini, S. Angular Optical Response of Cellulose Nanocrystal Films Explained by the Distortion of the Arrested Suspension upon Drying. Phys. Rev. Mater. 2019, 3 (4), 045601.

(9) Park, J. H.; Noh, J.; Schütz, C.; Salazar-Alvarez, G.; Scalia, G.; Bergström, L.; Lagerwall, J. P. F. Macroscopic Control of Helix Orientation in Films Dried from Cholesteric Liquid-Crystalline Cellulose Nanocrystal Suspensions. ChemPhysChem 2014, 15 (7), 1477-1484.

(10) Gray, D. G.; Mu, X. Chiral Nematic Structure of Cellulose Nanocrystal Suspensions and Films; Polarized Light and Atomic Force Microscopy. Materials 2015, 8 (11), 7873-7888.

(11) Saha, P.; Davis, V. A. Photonic Properties and Applications of Cellulose Nanocrystal Films with Planar Anchoring. ACS Appl. Nano Mater. 2018, 1 (5), 2175-2183.

(12) Pospisil, M. J.; Saha, P.; Abdulquddos, S.; Noor, M. M.; Davis, V. A.; Green, M. J. Orientation Relaxation Dynamics in Cellulose Nanocrystal Dispersions in the Chiral Liquid Crystalline Phase. Langmuir 2018, 34 (44), 13274-13282.

(13) Revol, J.-F. F.; Bradford, H.; Giasson, J.; Marchessault, R. H. H.; Gray, D. G. G. Helicoidal Self-Ordering of Cellulose Microfibrils in Aqueous Suspension. Int. J. Biol. Macromol. 1992, 14 (3), 170-172.

(14) Wang, P. X.; Hamad, W. Y.; MacLachlan, M. J. Structure and Transformation of Tactoids in Cellulose Nanocrystal Suspensions. Nat. Commun. 2016, 7, 11515.

(15) Tran, A.; Hamad, W. Y.; MacLachlan, M. J. Tactoid Annealing Improves Order in Self-Assembled Cellulose Nanocrystal Films with Chiral Nematic Structures. Langmuir 2018, 34 (2), 646-652.

(16) Zhao, T. H.; Parker, R. M.; Williams, C. A.; Lim, K. T. P.; FrkaPetesic, B.; Vignolini, S. Printing of Responsive Photonic Cellulose Nanocrystal Microfilm Arrays. Adv. Funct. Mater. 2019, 29 (21), 1804531.

(17) Mu, X.; Gray, D. G. Formation of Chiral Nematic Films from Cellulose Nanocrystal Suspensions Is a Two-Stage Process. Langmuir 2014, 30 (31), 9256-9260.

(18) Honorato-Rios, C.; Kuhnhold, A.; Bruckner, J. R.; Dannert, R.; Schilling, T.; Lagerwall, J. P. F. Equilibrium Liquid Crystal Phase Diagrams and Detection of Kinetic Arrest in Cellulose Nanocrystal Suspensions. Front. Mater. 2016, 3, 21.

(19) Parker, R. M.; Frka-Petesic, B.; Guidetti, G.; Kamita, G.; Consani, G.; Abell, C.; Vignolini, S. Hierarchical Self-Assembly of Cellulose Nanocrystals in a Confined Geometry. ACS Nano 2016, 10 (9), 8443-8449.

(20) Klockars, K. W.; Tardy, B. L.; Borghei, M.; Tripathi, A.; Greca, L. G.; Rojas, O. J. Effect of Anisotropy of Cellulose Nanocrystal Suspensions on Stratification, Domain Structure Formation, and Structural Colors. Biomacromolecules 2018, 19 (7), 2931-2943.

(21) Khan, M. J.; Khan, H. S.; Yousaf, A.; Khurshid, K.; Abbas, A. Modern Trends in Hyperspectral Image Analysis: A Review. IEEE Access 2018, 6, 14118-14129.
(22) Gao, L.; Smith, R. T. Optical Hyperspectral Imaging in Microscopy and Spectroscopy - A Review of Data Acquisition. J. Biophotonics 2015, 8 (6), 441-456.

(23) Medina, J. M.; Nascimento, S. M. C.; Vukusic, P. Hyperspectral Optical Imaging of Two Different Species of Lepidoptera. Nanoscale Res. Lett. 2011, 6, 369.

(24) Choi, S. H.; Byun, K. M. Naturally Occurring Order-Disorder Duality in Photonic Structures of the Haliotis Fulgens Abalone Shell. Opt. Mater. Express 2019, 9 (5), 2206-2215.

(25) Frka-Petesic, B.; Kelly, J. A.; Jacucci, G.; Guidetti, G.; Kamita, G.; Crossette, N. P.; Hamad, W. Y.; MacLachlan, M. J.; Vignolini, S. Retrieving the Coassembly Pathway of Composite Cellulose Nanocrystal Photonic Films from Their Angular Optical Response. Adv. Mater. 2020, 32 (19), 1906889.

(26) Mu, X.; Gray, D. G. Droplets of Cellulose Nanocrystal Suspensions on Drying Give Iridescent 3-D "Coffee-Stain" Rings. Cellulose 2015, 22 (2), 1103-1107.

(27) Klockars, K. W.; Yau, N. E.; Tardy, B. L.; Majoinen, J.; Kämäräinen, T.; Miettunen, K.; Boutonnet, E.; Borghei, M.; Beidler, J.; Rojas, O. J. Asymmetrical Coffee Rings from Cellulose Nanocrystals and Prospects in Art and Design. Cellulose 2019, 26 (1), 491-506.

(28) Colorimetry - Part 1: CIE Standard Colorimetric Observers; International Organization for Standardization: Geneva, Switzerland, 2007.

(29) Yeh, P. Optics of Anisotropic Layered Media: A New $4 \times 4$ Matrix Algebra. Surf. Sci. 1980, 96 (1-3), 41-53.

(30) Barboza, R.; Bortolozzo, U.; Clerc, M. G.; Residori, S. Berry Phase of Light under Bragg Reflection by Chiral Liquid-Crystal Media. Phys. Rev. Lett. 2016, 117, 053903.

(31) Ličen, M.; Majaron, B.; Noh, J.; Schütz, C.; Bergström, L.; Lagerwall, J.; Drevenšek-Olenik, I. Correlation between Structural Properties and Iridescent Colors of Cellulose Nanocrystalline Films. Cellulose 2016, 23 (6), 3601-3609.

(32) O'Keeffe, O.; Wang, P.-X.; Hamad, W. Y.; MacLachlan, M. J. Boundary Geometry Effects on the Coalescence of Liquid Crystalline Tactoids and Formation of Topological Defects. J. Phys. Chem. Lett. 2019, 10, 278-282.

(33) Revol, J.-F.; Godbout, L.; Dong, X.-M.; Gray, D. G.; Chanzy, H.; Maret, G. Chiral Nematic Suspensions of Cellulose Crystallites; Phase Separation and Magnetic Field Orientation. Liq. Cryst. 1994, 16 (1), 127-134.

(34) Van Bruggen, M. P. B.; Dhont, J. K. G.; Lekkerkerker, H. N. W. Morphology and Kinetics of the Isotropic-Nematic Phase Transition in Dispersions of Hard Rods. Macromolecules 1999, 32 (7), 22562264.

(35) Lettinga, M. P.; Kang, K.; Imhof, A.; Derks, D.; Dhont, J. K. G. Kinetic Pathways of the Nematic-Isotropic Phase Transition as Studied by Confocal Microscopy on Rod-Like Viruses. J. Phys.: Condens. Matter 2005, 17 (45), S3609-S3618.

(36) Elazzouzi, S. Self-Organization of Cellulose Whiskers Suspended in Water or in Apolar Organic Solvents, Ph.D. Thesis, 2006.

(37) Holmqvist, P.; Ratajczyk, M.; Meier, G.; Wensink, H. H.; Lettinga, M. P. Supersaturated Dispersions of Rodlike Viruses with Added Attraction. Phys. Rev. E - Stat. Nonlinear, Soft Matter Phys. 2009, 80, 031402.

(38) Honorato-Rios, C.; Lehr, C.; Schütz, C.; Sanctuary, R.; Osipov, M. A.; Baller, J.; Lagerwall, J. P. F. Fractionation of Cellulose Nanocrystals: Enhancing Liquid Crystal Ordering without Promoting Gelation. NPG Asia Mater. 2018, 10 (5), 455-465.

(39) Dong, X. M.; Revol, J.-F.; Gray, D. G. Effect of Microcrystallite Preparation Conditions on the Formation of Colloid Crystals of Cellulose. Cellulose 1998, 5 (1), 19-32.

(40) Revol, J.-F.; Godbout, D. L.; Gray, D. G. Solidified Liquid Crystals of Cellulose with Optically Variable Properties. US5629055A, 1997.

(41) Ni, R.; Belli, S.; Van Roij, R.; Dijkstra, M. Glassy Dynamics, Spinodal Fluctuations, and the Kinetic Limit of Nucleation in 
Suspensions of Colloidal Hard Rods. Phys. Rev. Lett. 2010, 105, 088302.

(42) Honorato-Rios, C.; Lagerwall, J. P. F. Interrogating Helical Nanorod Self-Assembly with Fractionated Cellulose Nanocrystal Suspensions. Commun. Mater. 2020, 1 (1), 69.

(43) Lettinga, M. P.; Kang, K.; Holmqvist, P.; Imhof, A.; Derks, D.; Dhont, J. K. G. Nematic-Isotropic Spinodal Decomposition Kinetics of Rodlike Viruses. Phys. Rev. E - Stat. Nonlinear, Soft Matter Phys. 2006, 73, 011412.

(44) Sztrum-Vartash, C. G.; Rabani, E. Lattice Gas Model for the Drying-Mediated Self-Assembly of Nanorods. J. Phys. Chem. C 2010, 114, 11040-11049.

(45) Cherpak, V.; Korolovych, V. F.; Geryak, R.; Turiv, T.; Nepal, D.; Kelly, J.; Bunning, T. J.; Lavrentovich, O. D.; Heller, W. T.; Tsukruk, V. V. Robust Chiral Organization of Cellulose Nanocrystals in Capillary Confinement. Nano Lett. 2018, 18 (11), 6770-6777.

(46) Frka-Petesic, B.; Radavidson, H.; Jean, B.; Heux, L. Dynamically Controlled Iridescence of Cholesteric Cellulose Nanocrystal Suspensions Using Electric Fields. Adv. Mater. 2017, 29 (11), 1606208.

(47) Kang, K.; Dhont, J. K. G. Glass Transition in Suspensions of Charged Rods: Structural Arrest and Texture Dynamics. Phys. Rev. Lett. 2013, 110 (1), 015901.

(48) Soga, I.; Dhinojwala, A.; Granick, S. Optorheological Studies of Sheared Confined Fluids with Mesoscopic Thickness. Langmuir 1998, $14,1156-1161$.

(49) Shafeiei-Sabet, S.; Hamad, W. Y.; Hatzikiriakos, S. G. Influence of Degree of Sulfation on the Rheology of Cellulose Nanocrystal Suspensions. Rheol. Acta 2013, 52, 741-751.

(50) Xu, Y.; Atrens, A.; Stokes, J. R. A Review of Nanocrystalline Cellulose Suspensions: Rheology, Liquid Crystal Ordering and Colloidal Phase Behaviour. Adv. Colloid Interface Sci. 2020, 275, 102076 .

(51) Boles, M. A.; Engel, M.; Talapin, D. V. Self-Assembly of Colloidal Nanocrystals: From Intricate Structures to Functional Materials. Chem. Rev. 2016, 116, 11220-11289.

(52) Lu, P. J.; Zaccarelli, E.; Ciulla, F.; Schofield, A. B.; Sciortino, F.; Weitz, D. A. Gelation of Particles with Short-Range Attraction. Nature 2008, 453, 499-504.

(53) Kamita, G.; Frka-Petesic, B.; Allard, A.; Dargaud, M.; King, K.; Dumanli, A. G. A. G.; Vignolini, S. Biocompatible and Sustainable Optical Strain Sensors for Large-Area Applications. Adv. Opt. Mater. 2016, 4 (12), 1950-1954.

(54) Berreman, D. W. Optics in Stratified and Anisotropic Media: 4 $\times$ 4-Matrix Formulation. J. Opt. Soc. Am. 1972, 62 (4), 502-510.

(55) Stallinga, S. Berreman $4 \times 4$ Matrix Method for Reflective Liquid Crystal Displays. J. Appl. Phys. 1999, 85, 3023.

(56) Castany, O. Python Implementation of Berreman's $4 \times 4$ Matrix Method, 2012.

(57) Bay, M. M.; Vignolini, S.; Vynck, K. PyLlama, version 1, 2020, https://github.com/VignoliniLab/PyLlama. 